\title{
Increasing indoor air quality by a natural sanitizing interior
}

\author{
Tetiana Tkachenko ${ }^{1 *}$ and Viktor Mileikovskyi ${ }^{2}$ \\ ${ }^{1}$ Department of Labor and Environmental Protection, Kyiv National University of Construction and \\ Architecture, Kyiv, 03037, Ukraine. \\ ${ }^{2}$ Heat Gas Supply and Ventilation Department, Kyiv National University of Construction and \\ Architecture, Kyiv, 03037, Ukraine.
}

\begin{abstract}
Low air quality is one of the most widespread reasons for diseases of respiratory, cardiovascular, immune, and nervous systems. The work aims to improve indoor air quality using the most natural method indoor phytoncide plants. R. Koch's sedimentation method has been used. The rule of V. L. Omelianskyi calculated the total viable number. Phytoncide activity was estimated by tests of leaf disks in Petri dishes with evenly seeded microbes. Researches in a high school showed a very high total viable number after studies - up to $6000 \mathrm{CFU} / \mathrm{cm} 3$. Tests of leaf disks allowed ranging the commonly used indoor plants by phytoncide activity. The most active large-size plants are Citrus limon and Ficus benjamina. From small-size plants, Azalea and Fuchsia were recommended. Amaryllis and Phoenix dactylifera have the lowest phytoncide activity. Final tests of indoor air showed the possibility of decreasing the total viable number in the high school rooms 2.5-3.5 times.
\end{abstract}

\section{Introduction}

Today people spend almost time inside the premises. In developed countries, the time of indoor stay is approximately $80 \%$ [1]. Therefore, the interior air quality is one of the most influencing factors on men's health [1-7], especially, on respiratory $[1,2,6]$, cardiovascular [1, 3, 6], immune [1] (incl. Allergy [7]) and nervous [8] systems. According to WHO, in 2016 4.2 million [5] of premature deaths are due to indoor air pollution. Outdoor air pollution causes 3.8 million [5]. $90 \%$ of people are affected by inadequate air quality [5].

Indoor air pollution can be classified to

- physical (heat [8-9], noise);

- chemical [5] (excessive moisture, tobacco smoke, formaldehyde, volatile organic compounds, carbon, sulfur and nitrogen oxides, ozone, asbestos particles, etc.);

- biological $[5,10]$ (horny particles, pollen, house dust mites, external allergens, various microorganisms, viruses, etc.).

The indoor concentration of air pollutants varies depending on the location, season, and daytime, the number and duration of people in a premise [11]. The most harmful pollutants

\footnotetext{
* Corresponding author: tkachenkoknuba@gmail.com
} 
are microorganisms and viruses. No microorganisms are living permanently in the air. The deficiency of moisture, nutrients, and solar radiation prevents reproduction. Microbes get into the air from soil and plants' surface, with production waste, from animal organisms, etc. When sneezing, coughing, and saying, there are many drops of mucus with epithelial cells and microorganisms from the upper respiratory tract released to the air. Airborne droplets form a stable microbial and viral aerosol, the fine fractions of which can penetrate even into the middle and lower parts of the human respiratory tract. The spread of microbes and viruses can be realized in another way, if the droplets ejected from the respiratory tract dry up on the surfaces and turn into bacterial dust. It has been proven that in a protein substrate, some bacteria survive longer, and such bacterial dust can move intensively with airflows. This can cause influenza, measles, tuberculosis, whooping cough, diphtheria, rubella, mumps, CoViD19 [10], allergy [7], etc.

Therefore, investigation and prevention of microbial air pollution are essential tasks. One option is to decrease microbial pollution in the natural biologic method of sanitizing interiors by introducing phytoncide plants. In our previous work [11], we proposed a photo design of sanitizing interiors for common areas of multi-floor buildings. Similar principles are useful for rooms of different purposes.

Plants have no active immune cells that can detect and kill intruders. They release phytoncides [12] - volatile organic substances that suppress pathogenic microbes around them. These substances are natural. They are present in forests, gardens, and parks. Therefore, most of them are not harmful to people or animals. Plants release the phytoncides continuously, which minimizing automatization. Another natural option is aromatic oils (Fig. 1) from phytoncide plants [12].

As shown in Fig. 1, a change of the concentration three times causes a change in microbes suppression by $5 \%$ except for Juniperus. High increase of the effectiveness between $\mathrm{c}=12.5$ $\mathrm{ul} / \mathrm{m} 3$ and $\mathrm{c}=15 \mathrm{ul} / \mathrm{m} 3$ was not explained in [12]. A blunder is possible.

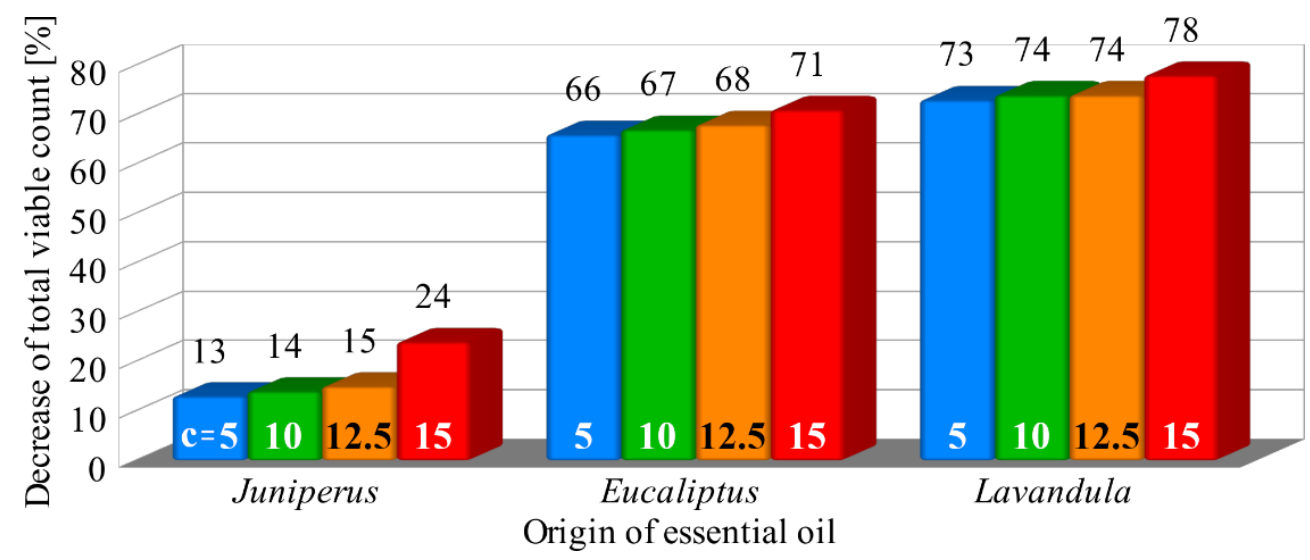

Fig. 1. Decrease of total viable number in indoor air by essential oils [12] $c$ - volume concentration of oil vapor $\left[\mathrm{ul} / \mathrm{m}^{3}\right]$.

If we neglect the last result, variation in effectiveness due to concentration will be comparable with experimental errors. Thus, the concentration can vary widely without a significant influence on the total viable number.

The method requires control of release during a day. The oils are expensive. They cause intensive odor, not everyone may like.

On the other hand, synthetic sanitizers are non-natural. They can be harmful or organoleptic, especially at a continuous impact on the respiratory system. Thus, the phytoncide plants' 
introduction is the most natural, easy, cheap, and human-friendly method of sanitizing interiors.

\section{Method}

\subsection{A method for determining the microbial content in indoor air}

The objects of research of air microflora were a library (10 man-hours $\left./ \mathrm{m}^{2}\right)$, a lecture hall (30 man-hours $\left./ \mathrm{m}^{2}\right)$, a sports hall $\left(25\right.$ man-hours $\left./ \mathrm{m}^{2}\right)$, a laboratory $\left(10 \mathrm{man}\right.$-hours $\left./ \mathrm{m}^{2}\right)$, and a warehouse (reference room, no people) in a high school, where students study a total of more than 8,000 people.

This study of air microflora was performed by the sedimentation method by R. Koch [13, 14]. This method is the simplest and the most accessible. Petri dishes of area $A\left[\mathrm{~cm}^{2}\right]$ with sterile nutrient medium should be located in different places of a room and opened for 5 minutes. Dust particles with microbes settle on the surface of nutrient agar.

At the end of the exposure, the cups should be closed, marked indicating the date/time and the corresponding measurement point, and placed in a thermostat at a temperature of 28 $30{ }^{\circ} \mathrm{C}$ for two days.

During this time, the number of colonies $n$ of microorganisms should be counted, and growth on agar plates must be investigated. For calculation of the number of microorganisms, it is possible to use the rule of V. L. Omelianskyi [12]: all microbes and their spores that are contained in $10 \mathrm{dm}^{3}$ of air, settle on $100 \mathrm{~cm}^{2}$ area during 5 minutes. Thus, the number of microbes in one cubic meter of air.

$$
N=10000 n / A ., \mathrm{m}^{-3}
$$

After that, the cultural properties of the most characteristic colonies should be studied. For this, preparations will be made of them. The preparations will be examined under a microscope, and the genus will be established based on morphological and cultural features. In some species, it is possible to identify bacteria using albums-determinants.

\subsection{Method of determining the phytoncide activity}

The objects of the study were the following indoor plants:

- azalea (Azalea),

- amaryllis (Amaryllis),

- room lemon (Citrus limon),

- date palm (Phoenix dactylifera),

- Chinese rose (Hibiscus rosa-sinensis),

- fuchsia (Fuchsia),

- ficus benjamina (Ficus benjamina).

Disks of $1 \mathrm{~cm}$ from the leaves of the studied plants should be cut and chemically sterilized. Continuous sowing of the studied microorganism spores should be performed in two Petri dishes on a solidified wort agar surface. To do this, by a sterile pipette, $0.2 \mathrm{~cm}^{3}$ of suspension of spores should be taken and pour on the surface of the agar. It should be distributed carefully and evenly over the entire surface by Drygalskyi spatula.

After that, the plant discs should be put in one dish. The second dish with wort agar will be used as a reference one. After completing the seeding procedure, the cups should be kept in a thermostat at $25^{\circ} \mathrm{C}$ for fungi and $30^{\circ} \mathrm{C}$ for bacteria. Lysis zones will indicate plant 
phytoncides' effect on the studied fungus (or bacteria) without microorganisms. Such zones should be measured [15].

All experiments were repeated three times. The obtained experimental data were subjected to analysis of variance. It allows obtaining average values and the probability of the influence of factors (difference in the level of total viable count in the premises).

\section{Results and discussion}

\subsection{The microbial content in indoor air}

he research results in colony-forming units per square centimeter are in Fig. 2 and Fig. 3. In Fig. 4, there are colonies on Petri dishes, exposed before and after studies.

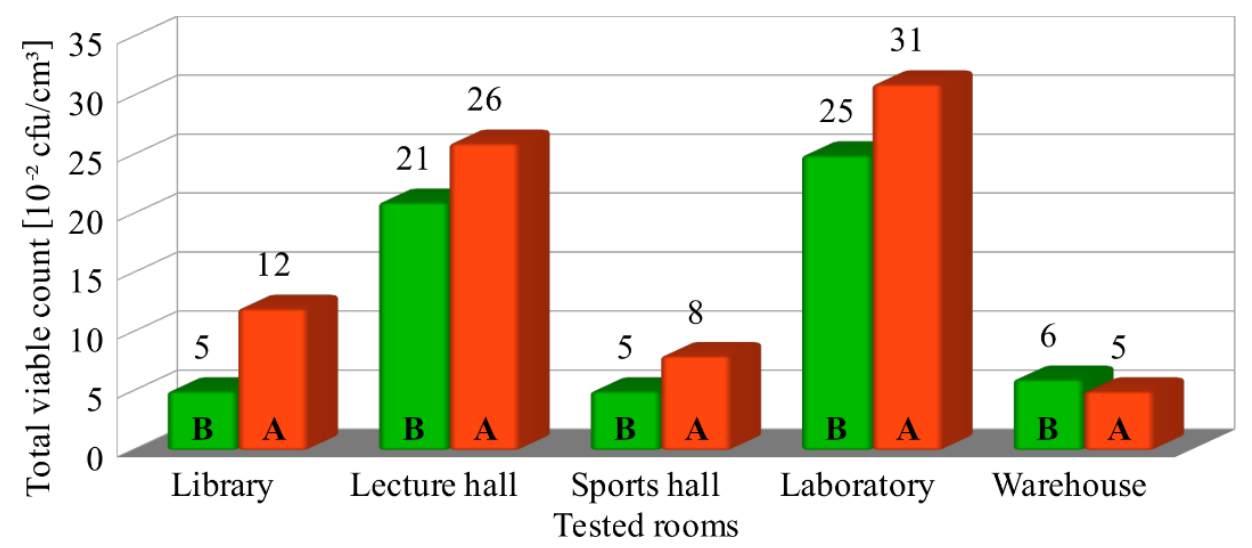

Fig. 2. Average total viable count in indoor air in autumn (Author's Analysis, 2020) $\mathrm{B}$ - before studies; A - after studies.

The results show a trustworthy increase in the number of microbes after studies. In the library, lecture hall, and laboratory, the increase is more significant than exceeding sanitary norms. A possible reason is a transfer of microbes on outer clothing. In the sports hall, the number of bacteria rises slightly in autumn and does not rise in winter. It is due to changing clothes to gym suits, which have minimum contact with bacteria in the environment.

The warehouse has minimum bacteria before and after studies because of the minimum people access. In the laboratory, there was a growth of some mold fungi. It can indicate facts of ingestion. It is shown that air pollution by dust, smoke, soot, etc. increases microorganisms.

The data from Finland schools [16] is better. In classes of Helsinki and Vantaa schools, the total viable count is $0-540 \mathrm{CFU} / \mathrm{m}^{3}$, except for a class with $1680 \mathrm{CFU} / \mathrm{m}^{3}$. In Italy [17], the situation is worse. 


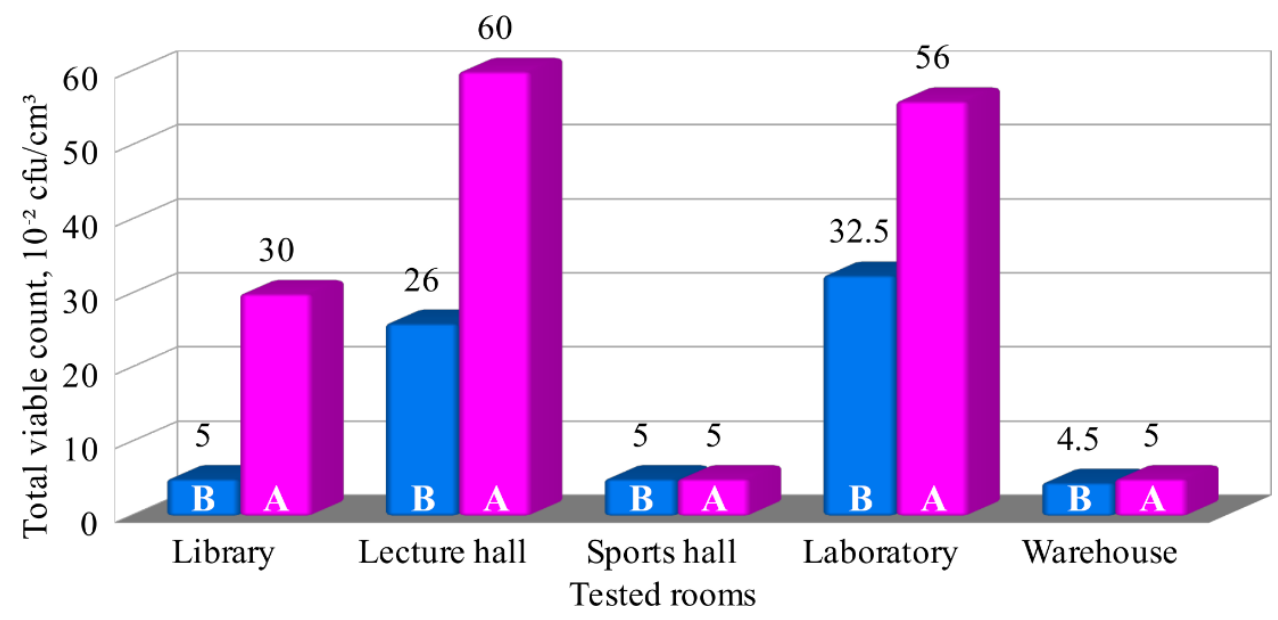

Fig. 3. Average total viable count in indoor air in winter (Author's Analysis, 2020):

$\mathrm{B}$ - before studies; A - after studies.
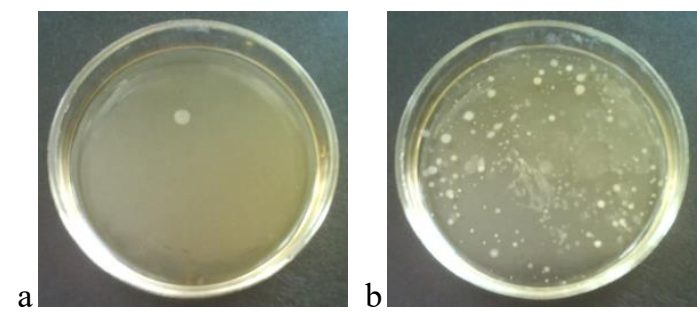

Fig. 4. Grown colonies in Petri dishes (Author's Analysis, 2020): a - exposed before studies; b exposed after studies.

In offices and libraries, the total viable count reaches, accordingly, 8860 and 1265 $\mathrm{CFU} / \mathrm{m}^{3}$. In the libraries of Jimma University in Ethiopia [18], the number is 367-2595 $\mathrm{CFU} / \mathrm{m}^{3}$. Therefore, microbial pollution is intermediate compared to world data. The most often spores of microbes are Bacillus, Clostridium, conidia of fungi, pieces of mycelium, cysts of protozoa and unicellular aquatic plants, pollen, micrococcus, sarcina, Bac. subtilis, Bac. megaterium, Bac. cereus, Actinomycetes, mold fungi, yeast fungi (Saccharomyces), etc.

It is known that in forests, especially coniferous, the number of microbes is lower because of phytoncides. Green plantations release phytoncides and absorb dust with microbes by foliage. This allows using the most natural method of bacteria control - sanitizing green designs of interiors. Green [19] structures (roofs, walls, terraces) are also helpful for suppressing bacteria in the air around buildings. To check the effectiveness, the next stage of research will focus on plants' phytoncide properties on green structures.

\subsection{The phytoncide activity of indoor plants}

\subsubsection{Tests of leaf disks}

When studying the microflora, yeast fungi of the genus Saccharomyces represented a significant amount of biological contamination. We used them as a test culture. Yeast is not a pathogenic or opportunistic organism used in research conducted in scientific laboratories 
of educational institutions. The research of suppression of the fungi by leaf disks (Fig. 5, 6) shows the phytoncide activity of all of the studied plants.
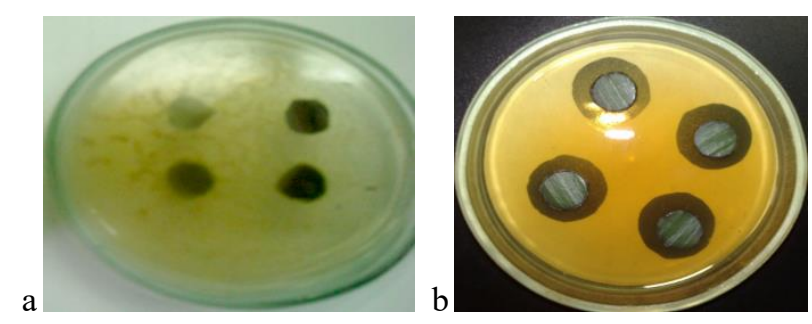

Fig. 5. Testing leaf disks in Petri dishes (Author's Analysis, 2020): $a$ - setting the test; $b$ - an example of test results for Amaryllis.

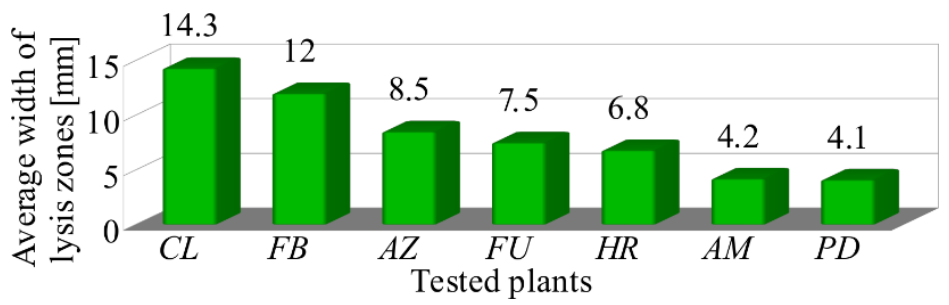

Fig. 6. Results of testing leaf disks in Petri dishes (Author's Analysis, 2020).

The most significant inhibition of yeast fungi was observed at the introduction leaves disks of Citrus limon. Ficus benjamina is slightly weaker than lemon in its phytoncide activity, which may be due to the release of other biologically active substances from the fleshy leaves of this plant. After them, there are Azalea, Fuchsia, and Hibiscus rosa-sinensis. The leaves of Amaryllis and Phoenix dactylifera caused the weakest phytoncide effect. The plants release phytoncides to the ambient air. The effect of them on the premises should be tested by seeding the air microflora with and without plants.

\subsubsection{Tests of air microflora of indoor air with and without plants}

The final tests are performed by the method described in Chapter 2.1. We found that the presence of plants in the room has a bactericidal effect on the air microflora. The study revealed a significant (2.5-3.5 times) reduction in the number of microorganisms in the air of the studied premises under houseplants' influence (Fig. 7).

The researches show the effectiveness of using plants as the most natural method of design the sanitizing interiors. We recommend using room lemon (Citrus limon) and ficus benjamina (Ficus benjamina) because they grow up to large sizes and have very high activity. As the second choice, we can use large-sized plants - Chinese rose (Hibiscus rosa-sinensis), which is from 1.76 times to 2.10 times weaker (Fig. 6). For plants of small size, we recommend beautifully flowering plants - azalea and fuchsia.

As it is shown in Fig. 7, such plants significantly decrease indoor microbial pollution. It is not possible to avoid an external air supply. However, this can partially compensate for the decrease in air exchange caused by the energy efficiency norms. It is essential during the time of smog or strong traffic jams when the supply of external air is impossible. 

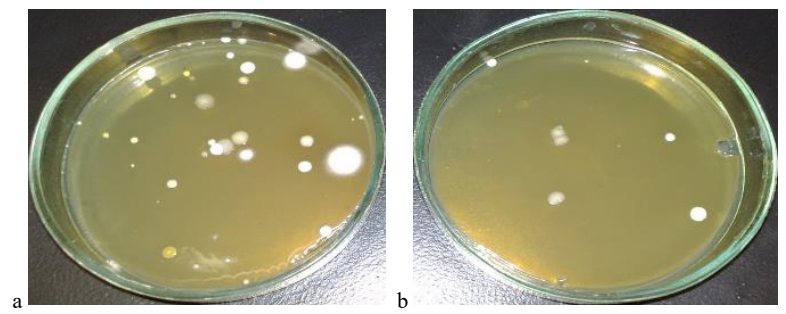

Fig. 7. Petri dishes after exposition in the room (Author's Analysis, 2020): a - without plants, b with plants.

\section{Conclusion}

Microbes strongly pollute indoor air in educational rooms of a high school. After studies, pollution can exceed norms. The average total viable count can approach $6000 \mathrm{CFU} / \mathrm{cm} 3$. One of the most natural solutions is using indoor phytoncide plants. We propose using largesized plants - room lemon (Citrus limon) and ficus benjamina (Ficus benjamina) - combined with small-sized ones - Azalea and Fuchsia. The plants should be put in each premise in places with appropriate illumination without creating obstacles at evacuation paths. The number of recommended plants should be as much as possible to ensure the maximum effect. In large auditoriums, some plants can be hung on walls or set into places with inappropriate insolation. In such conditions, local illumination by phytolamps is necessary. LED ones are the most energy-effective. To avoid students' distraction, the illumination can be switched off during studies and switched on during the absence of them and breaks. In this case, fluorescent lamps are the worst solution because of wear when switching and, as a result, additional mercury environment pollution during utilization. Experimental research shows the high phytoncide activity of the plants. They can 2.5-3.5 times decrease the total viable count.

\section{References}

1. L. Ferguson, J. Taylor, M. Davies, C. Shrubsole, P. Symonds, S. Dimitroulopoulou, Environ. Int, 143, 105748 (2020) https://doi.org/10.1016/j.envint.2020.105748

2. Xue-Yan Zheng, Hong Ding, Li-Na Jiang, Shao-Wei Chen, Jin-Ping Zheng, Min Qiu, Ying-Xue Zhou, Qing Chen, Wei-Jie Guan, PLoS One, 10, e0138146 (2015)

3. F. Kelly, R. Maynard The Effects of Long-Term Exposure to Ambient Air Pollution on Cardiovascular Morbidity: Mechanistic Evidence: A report by the Committee on the Medical Effects of Air Pollutants (2018)

4. Chong Wang, Sung-Jun Yoo, Shin-Ichi Tanabe, Kazuhide Ito, EBE, 1, 423 (2020)

5. S. D. Lowther, Wei Deng, Zheng Fang, D. Booker, D. J. Whyatt, O. Wild, Xinming Wang, K. C. Jones, Environ. Int., 144, 106001 (2020)

6. R. A. Sharpe, K. E. Machray, L. E. Fleming, T. Taylor, W. Henley, T. Chenore I. Hutchcroft, J. Taylor, C. Heaviside, B. W. Wheeler, Environ. Int., 133 (2019)

7. E. Kochelaev, S. Shkarednaia, T. Zyrianova, Stroitelnyie materialy, 133, 105164 (2019)

8. P. V. Moulton, Wei Yang, Journal of Environmental and Public Health, 2012, 472751 (2012) 
9. C. A. Jensen, X. Cadorel A. Chu, Ventilation for reduced heat stress in apartments, in Back to the Future: The Next 50 Years: 51-st International Conference of the Architectural Science Association (ANZAScA), CEE 2019, 29 November - 132 December 2017, Wellington, New Zealand, 615 (2017)

10. G Buonanno, L. Morawska, L. Stabile, Environ. Int, 145 (2020)

11. T. Tkachenko, V. Mileikovskyi, Solution of sick building syndrome problem using indoor plants, in Environmental Innovations: Advances in Engineering, Technology and Management, EIAETM 2019, 23-27 September 2019, P - ESEM, 6, 405 (2019)

12. T. Bilyk, N. Lukianenko, K. Vityk, O. Havryliuk, Proceedings of NAU, 75 (2018)

13. D. Diaconu, M. Tatarciuc, D. Tatarciuc, A. Vitalariu, RJOR, 4 (2012)

14. E. R. Cernei, D. C. Maxim, R. Mavru, L. L. Indrei, RJOR, 5 (2013)

15. L. A. Vinogradova, Indication of biocenosis of potentially pathogenic, indicator and pathogenic bacteria in water bodies of the environment, in Methods for indication of biocenosis of pathogenic and potentially pathogenic microorganisms in environmental objects, Moscow, MNIIG (1985)

16. C. Vornanen-Winqvist, K. Jarvi, M. A. Andersson, C. Duchaine, V. Letourneau, O. Kedves, L. Kredics, R. Mikkola, J. Kurnitski, H. Salonen, Environ. Int., 141, 105781 (2020)

17. P. Grisoli, M. Albertoni, M. Rodolfi, Appl. Sci., 9, 1101 (2019)

18. S. F. Hayleeyesus, A. M. Manaye, Asian Pac. J. Trop. Biomed., 4, S312 (2014)

19. T. Tkachenko, V. Mileikovskyi, SJST, 42, 50 (2020) 